\title{
Feasibility and safety of left atrial posterior wall isolation with a new Cryoballoon technology in patients with persistent atrial fibrillation
}

Antonio Bisignani ${ }^{1}$, Luigi Pannone ${ }^{2}$, Vincenzo Miraglia ${ }^{3}$, Juan Sieira ${ }^{4}$, Saverio Iacopino ${ }^{5}$, Gezim Bala ${ }^{3}$, Erwin Stroker ${ }^{6}$, Ingrid Overeinder ${ }^{7}$, Alexandre Almorad ${ }^{3}$, Anaïs Gauthey ${ }^{3}$, Cinzia Monaco ${ }^{3}$, Gaetano Paparella ${ }^{8}$, Thiago Guimarães Osório ${ }^{8}$, Antonio Sorgente ${ }^{9}$, Luc Jordaens $^{10}$, Pedro Brugada ${ }^{11}$, Gian battista Chierchia ${ }^{12}$, and Carlo de Asmundis ${ }^{13}$

${ }^{1}$ Policlinico Universitario Agostino Gemelli

${ }^{2}$ IRCCS Ospedale San Raffaele

${ }^{3}$ Vrije Universiteit Brussel

${ }^{4}$ UZ Brussel

${ }^{5}$ Villa Maria Cecilia

${ }^{6}$ Universitair Ziekenhuis Brussel

${ }^{7}$ UZ Brussel, Vrije Universiteit Brussel

${ }^{8}$ Heart Rhythm Management Center, Cardiovascular Center UZ Brussel Vrij Universiteit

Brussel, Dpt Cardiology laarbeeklaan 101 Brussel, Jette, BE 1090 0032-2-4763038,

Cardiology Brussels, BE 0032-2-4763038

${ }^{9}$ Cleveland Clinic Abu Dhabi

${ }^{10}$ Erasmus MC

${ }^{11}$ Free University of Brussels

${ }^{12}$ heart rhythm management center

${ }^{13}$ UZ Brussel-VUB

November 16, 2021

\begin{abstract}
Introduction: Left atrial posterior wall isolation (LAPWI) is often performed in addition to pulmonary vein isolation (PVI) to improve outcome in patients with persistent atrial fibrillation (AF). In this setting, LAPWI + PVI using cryoballoon ablation had comparable results with radiofrequency ablation (RFA). The aim of the study is to evaluate the feasibility and safety of a new cryoballoon ablation system in PVI + LAPWI isolation, comparing it with the historical platform. Methods: The study was a prospective, non-randomized, single center study. Forty consecutive patients, undergoing PVI + LAPWI with the novel POLARx, were compared to 40 consecutive patients who underwent the same procedure with the established Arctic Front Advance PRO. Results: Acute isolation was achieved in all PVs in both groups and LAPWI was achieved in 38 patients (95\%) in the POLARx group and in 36 patients (90\%) in Arctic Front group. Procedural outcomes were similar between the two groups, except for lower temperatures during cryoapplications in the POLARx group, for both PVI and LAPWI. The complication rate was low and similar between groups. Conclusion: LAPWI+PVI with the novel POLARx Cryoballoon is feasible and safe; the results are comparable with the Arctic Front Advance PRO system.
\end{abstract}

\section{Hosted file}


Manuscript.docx available at https://authorea.com/users/446525/articles/545730-feasibilityand-safety-of-left-atrial-posterior-wall-isolation-with-a-new-cryoballoon-technology-inpatients-with-persistent-atrial-fibrillation

\section{Hosted file}

Table.docx available at https://authorea.com/users/446525/articles/545730-feasibility-andsafety-of-left-atrial-posterior-wall-isolation-with-a-new-cryoballoon-technology-inpatients-with-persistent-atrial-fibrillation

\section{Hosted file}

Figure.docx available at https://authorea.com/users/446525/articles/545730-feasibility-andsafety-of-left-atrial-posterior-wall-isolation-with-a-new-cryoballoon-technology-inpatients-with-persistent-atrial-fibrillation 